\title{
The broad-band spectrum of Cygnus X-1 measured by INTEGRAL
}

\author{
M. Cadolle Bel ${ }^{1,2}$, P. Sizun ${ }^{1}$, A. Goldwurm ${ }^{1,2}$, J. Rodriguez ${ }^{1,3,4}$, P. Laurent ${ }^{1,2}$, A. A. Zdziarski ${ }^{5}$, L. Foschini ${ }^{6}$, \\ P. Goldoni ${ }^{1,2}$, C. Gouiffès ${ }^{1}$, J. Malzac ${ }^{7}$, E. Jourdain ${ }^{7}$, and J.-P. Roques ${ }^{7}$
}

\author{
1 Service d'Astrophysique, CEA-Saclay, 91191 Gif-Sur-Yvette, France \\ e-mail: mcadolle@cea.fr \\ 2 APC-UMR 7164, 11 place M. Berthelot, 75231 Paris, France \\ 3 AIM-UMR 7158, France \\ 4 ISDC, 16 Chemin d'Ecogia, 1290 Versoix, Switzerland \\ 5 N. Copernicus Astronomical Center, 00-716 Warsaw, Poland \\ ${ }^{6}$ INAF/IASF, Sezione di Bologna, Via Gobetti 101, 40129 Bologna, Italy \\ 7 Centre d'Étude Spatiale des Rayonnements, 31028 Toulouse, France
}

Received 15 March 2005 / Accepted 31 August 2005

\section{ABSTRACT}

The INTEGRAL satellite extensively observed the black hole binary Cygnus X-1 from 2002 November to 2004 November during calibration, open time and core program (Galactic Plane Scan) observations. These data provide evidence for significant spectral variations over the period. In the framework of the accreting black hole phenomenology, the source was most of the time in the Hard State and occasionally switched to the so-called "Intermediate State". Using the results of the analysis performed on these data, we present and compare the spectral properties of the source over the whole energy range $(5 \mathrm{keV}-1 \mathrm{MeV})$ covered by the high-energy instruments on board INTEGRAL, in both observed spectral states. Fe line and reflection component evolution occurs with spectral changes in the hard and soft components. The observed behaviour of Cygnus X-1 is consistent with the general picture of galactic black holes. Our results give clues to the physical changes that took place in the system (disc and corona) at almost constant luminosity during the spectral transitions and provide new measures of the spectral model parameters. In particular, during the Intermediate State of 2003 June, we observe in the Cygnus X-1 data a high-energy tail at several hundred $\mathrm{keV}$ in excess of the thermal Comptonization model which suggests the presence of an additional non-thermal component.

Key words. black hole physics - stars: individual: Cygnus X-1 - gamma rays: observations - X-rays: binaries - X-rays: general

\section{Introduction}

Galactic Black Holes (BH) X-ray binary systems display highenergy emission characterized by spectral and flux variabilities on time scales ranging from milliseconds to months. These systems are generally found in two major states mainly defined by the relative variable contributions of soft and hard $\mathrm{X}$-ray components, radio spectral properties and timing behaviour (McClintock \& Remillard 2003; Nowak 2002). In the Hard State (HS), the X-ray and $\gamma$-ray spectrum is generally described by a power law model with an exponential cutoff at a few hundred $\mathrm{keV}$, accompanied by relevant radio emission; it can be modeled by thermal Comptonization of cool seed photons in a hot electron plasma (Gierliński et al. 1997; Dove et al. 1998). The soft ( 0.1-2 keV) black body component is very weak or too soft to contribute significantly. The Thermal Dominant State (TDS) instead shows a strong thermal component with a characteristic temperature of at most a few $\mathrm{keV}$ that dominates the $\mathrm{X}$-ray spectrum. No, or very weak and spectrally steep, hard X-ray emission is observed; the radio emission is quenched or very faint. This spectrum is generally associated with a geometrically thin and optically thick accretion disc (Shakura \& Sunyaev 1973). In addition to these two canonical states, other states have been identified, characterized either by an even greater luminosity than in the TDS (the "Steep Power law State") or by variability and X-ray spectral properties mostly intermediate between the HS and the TDS (Belloni et al. 1996): the "Intermediate State" (IS).

Cygnus X-1/HDE 226868 is one of the first X-ray binaries detected; it belongs to the $\mathrm{BH}$ binary category. Among the brightest X-ray sources of the sky, it is also very variable on different time scales. The assumption that Cygnus X-1 ranks among the microquasars has been confirmed by the detection of a relativistic jet (Stirling et al. 2001). Since its discovery in 1964 (Bowyer et al. 1965), it has been extensively observed as the prototype of $\mathrm{BH}$ candidates in radio/optical wavelengths and with all high-energy instruments, from soft X-rays to $\gamma$-rays, e.g., with ASCA (Gierliński et al. 1999), SIGMA (Salotti et al. 1992), RXTE (Dove et al. 1998; Pottschmidt et al. 2003a), BeppoSAX (Frontera et al. 2001; Di salvo 2001) and CGRO (McConnell et al. 2000, 2002). This persistent source, located at $\sim 2.4 \pm 0.5 \mathrm{kpc}$ 
(McClintock \& Remillard 2003, Table 4.1), accretes via a strong stellar wind coming from its companion, a O9.7I star of $20 M_{\odot}$ (Ziólkowski 2005) with an orbital period of 5.6 days. The mass function constrains the inclination angle of the system between $25^{\circ}$ and $67^{\circ}$ (Gierliński et al. 1999) and we adopted the value of $45^{\circ}$.

Cygnus X-1 spends most of its time (90\% until 1998 see, e.g., Gierliński et al. 1999) in the HS, with a relatively low flux in soft $\mathrm{X}$-rays $(\sim 1 \mathrm{keV})$ and a high flux in hard $\mathrm{X}$-rays $(\sim 100 \mathrm{keV})$. Its spectrum is roughly described by a power law with a photon index $\Gamma$ between $1.4-2.1$; a break at energies higher than $\sim 50 \mathrm{keV}$ is present. This state is also characterized by a large timing variability. Occasionally, the source switches to the TDS with $\Gamma>2.3$. During 1996 June, in addition to the dominant black body component and the hard component, a high-energy tail extending up to $10 \mathrm{MeV}$ was discovered (McConnell et al. 2002). In this state little variability is observed. The IS, in which the source exhibits a relatively soft hard X-ray spectrum ( $\Gamma \sim 2.1-2.3)$ and a moderately strong soft thermal component (Méndez \& van der Klis 1997), often appears when the source is about to switch from one main state to another. When not associated with a clear spectral transition, this state is called a "Failed State Transition" (FST). In the IS, the source displays a complicated pattern of timing properties. In the past few years, the source has been deeply observed in the IS and in the TDS (Zdziarski et al. 2002; Pottschmidt et al. 2003a; Gleissner et al. 2004a; Zdziarski \& Gierliński 2004). In addition to the thermal and power law components, other spectral features can be present in the spectrum: a reflection component peaking around $30 \mathrm{keV}$ and, most noticeably, a Fe $\mathrm{K} \alpha$ line and Fe edge between 6 and $7 \mathrm{keV}$. These features can be visible in both spectral states.

The INTErnational Gamma-Ray Astrophysics Laboratory (INTEGRAL) mission (Winkler et al. 2003) is an European Space Agency satellite launched on 2002 October 17, carrying two main $\gamma$-ray instruments, IBIS (Ubertini et al. 2003) and SPI (Vedrenne et al. 2003), and two X-ray monitors JEM$\mathrm{X}$ (Lund et al. 2003). Composed of two detectors, ISGRI (Lebrun et al. 2003) and PICsIT (Di Cocco et al. 2003), the IBIS coded mask instrument covers the energy range between $15 \mathrm{keV}$ and $10 \mathrm{MeV}$. The SPI telescope works in the $20 \mathrm{keV}-8 \mathrm{MeV}$ range and the JEM-X monitors provide spectra and images in the nominal 3-35 keV band. As a bright hard $\mathrm{X}$-ray source, Cygnus $\mathrm{X}-1$ is a prime target for INTEGRAL and has been extensively observed during the Performance Verification (PV) Phase of the mission, when the source was in the HS (Bazzano et al. 2003; Bouchet et al. 2003; Cadolle Bel et al. 2004; Pottschmidt et al. 2003b). Pottschmidt et al. (2005) also reported on preliminary analysis of the high time-resolution Galactic Plane Scan (GPS) observations of Cygnus X-1 (up to 2004 April) in the $15-70 \mathrm{keV}$ range. In the present work, we report the results over a wide energy band (from $5 \mathrm{keV}$ up to $1 \mathrm{MeV}$ ) of several sets of observations of Cygnus X-1, including part of the PV-Phase observations not yet exploited, the first observations of Cygnus X-1 in the Open Time program, a larger amount of GPS data than previously analyzed and the data from the 2004 November calibration period. For the first time, up to $1.5 \mathrm{Ms}$ of INTEGRAL data of Cygnus X-1, collected over two years from 2002 November to 2004 November, are presented, fully exploiting the broad-band capability of all high-energy instruments of the mission.

\section{Observations and data reduction}

Table 1 reports the general periods (epochs) of the observations used, giving for each of them the instrument data available, date range, exposure (per instrument) and observing modes. Epoch 1 includes part of the PV-Phase observations of Cygnus $\mathrm{X}-1$. To discuss the time evolution of the source, we report here the IBIS/ISGRI light curves and hardness ratios obtained during most of the PV-Phase observations of Cygnus X-1 (first line of Table 1), but since spectral results were presented in previous works, we studied more specifically the broad-band spectrum (using JEM-X, IBIS and SPI data) only for those PVPhase observations not yet fully exploited, i.e., those performed between 2002 December 9-11 (epoch 1). The Open Time observation was performed on 2003 June 7-11 (epoch 2) with a $5 \times 5$ dither pattern (Jensen et al. 2003). The effective exposure time was $275 \mathrm{ks}$ for JEM-X2, $292 \mathrm{ks}$ for IBIS and $296 \mathrm{ks}$ for SPI. For this latter period, preliminary results can be found in Malzac et al. (2004) and Cadolle Bel et al. (2004) but we report here the complete study of the average spectrum while, in a future work, Malzac et al. (2005, submitted) will present the variability properties of the source. Epoch 3 and epoch 4 refer respectively to the set of Cygnus X-1 observations during the core program GPS and the 2004 November calibrations. Unfortunately, for the short interrupted GPS exposures, the SPI data are unusable because a sensitive evaluation of the background is not possible: only JEM-X and IBIS/ISGRI data are used. The periods of our different INTEGRAL observations, presented in Table 1, are also indicated in Fig. 1 (epochs from numbers 1 to 4 ).

We reduced the IBIS and JEM-X data with the standard analysis procedures of the Off-Line Scientific Analysis OSA 4.2 released by the ISDC, whose algorithms are described in Goldwurm et al. (2003) and Westergaard et al. (2003) for IBIS and JEM-X respectively. A basic selection was performed to exclude those pointings too close to radiation belt entry or exit, or spoilt by too much noise. To avoid uncertainties in the response files for high off-axis angles, we also selected the IBIS data of the observations for which the source was in the fully coded field of view, i.e., with an offset from the telescope axis no larger than $4.5^{\circ}$, and JEM-X data for maximum offset angles of $3^{\circ}$. Following recommendations of the OSA 4.2 release, IBIS/ISGRI events were selected to have corrected energies $>20 \mathrm{keV}$ and rise time channels between 7 and 80 . For the background correction, we used a set of IBIS/ISGRI maps derived in 256 energy channels from empty field observations (these maps will be the default IBIS/ISGRI correction maps for the OSA 5.0 release) combined with our chosen energy bins while, for the off-axis correction maps and the response matrices, we used those of the official OSA 4.2 release. In the analysis, we considered the presence of the two other sources of the region, Cygnus X-3 and EXOSAT 2030+375, when they 
Table 1. Log of the Cygnus X-1 observations analyzed in this paper.

\begin{tabular}{cclcc}
\hline \hline Epoch & Instrument & $\begin{array}{l}\text { Observation Period } \\
(\text { date yy/mm/dd) }\end{array}$ & $\begin{array}{c}\text { Exposure } \\
(\mathrm{ks})\end{array}$ & $\begin{array}{c}\text { Observation } \\
\text { Type }\end{array}$ \\
\hline & IBIS & $02 / 11 / 25-02 / 12 / 15$ & 810 & staring, $5 \times 5^{a}$, hex $^{b}$ \\
1 & IBIS/SPI/JEM-X & $02 / 12 / 09-02 / 12 / 11$ & $365 / 365 / 31$ & $5 \times 5$ \\
2 & IBIS/SPI/JEM-X & $03 / 06 / 07-03 / 06 / 11$ & $292 / 296 / 275$ & $5 \times 5$ \\
3 & IBIS/JEM-X & $03 / 03 / 24-04 / 09 / 10$ & $269 / 35$ & GPS $^{c}$ \\
4 & IBIS/SPI/JEM-X & $04 / 11 / 22$ & $8 / 8 / 6$ & calibration \\
\hline
\end{tabular}

Notes:

a $5 \times 5$ dither pattern around the nominal target location.

${ }^{b}$ Hexagonal pattern around the nominal target location.

${ }^{c}$ Individual exposures separated by $6^{\circ}$ along the scan path, shifted by $27.5^{\circ}$ in galactic longitude.

(Observations indicated on the first line also used, together with epochs 1-4, for Figs. 2 and 3.)

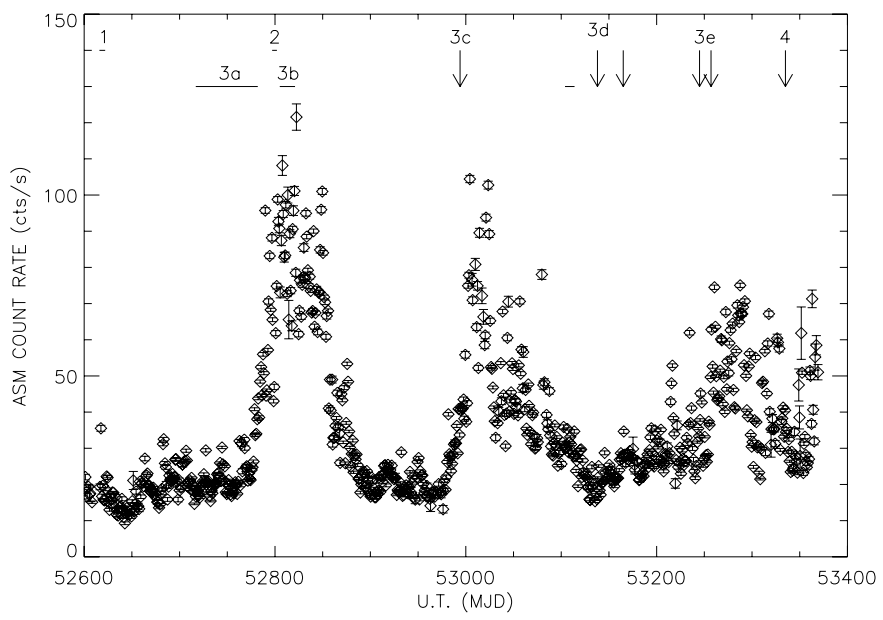

Fig. 1. RXTE/ASM daily average (1.5-12 keV) light curve of Cygnus X-1 from 2002 November to 2004 November $(\mathrm{MJD}=\mathrm{JD}-2400000.5)$ with the periods of our INTEGRAL observations (see text and Tables 1 and 2 for epoch definitions).

were active. For the IBIS/ISGRI spectral extraction however, we implemented the most recent module (prepared for the OSA 5.0 delivery) which is based on the least squares fit done on background and efficiency corrected data, using coded source zones only. This option minimizes spurious features in the extracted spectra, which appear in particular when the sources are weak, partially coded and the background poorly corrected (A. Gros, private communication). For the PICsIT spectral extraction, we took the flux and error values in the mosaic image at the best-fit position found for the source. We used the response matrices officially released with OSA 4.2, rebinned to the 8 energy channels of the imaging output.

The SPI data were preprocessed with OSA 4.2 using the standard energy calibration gain coefficients per orbit and excluding bad quality pointings which have anomalous exposure and dead time values (or with a high final $\chi^{2}$ during imaging). The spiros 9.2 release (Skinner \& Connell 2003) was used to extract the spectra of Cygnus X-1, Cygnus X-3 and EXO $2030+375$, with a background model proportional to the saturating event count rates in the Ge detectors. Concerning the instrumental response, version 15 of the IRF (Image Response Files) and version 2 of the RMF (Redistribution Matrix Files) were used for epoch 1 and 2, e.g., prior to detector losses, while

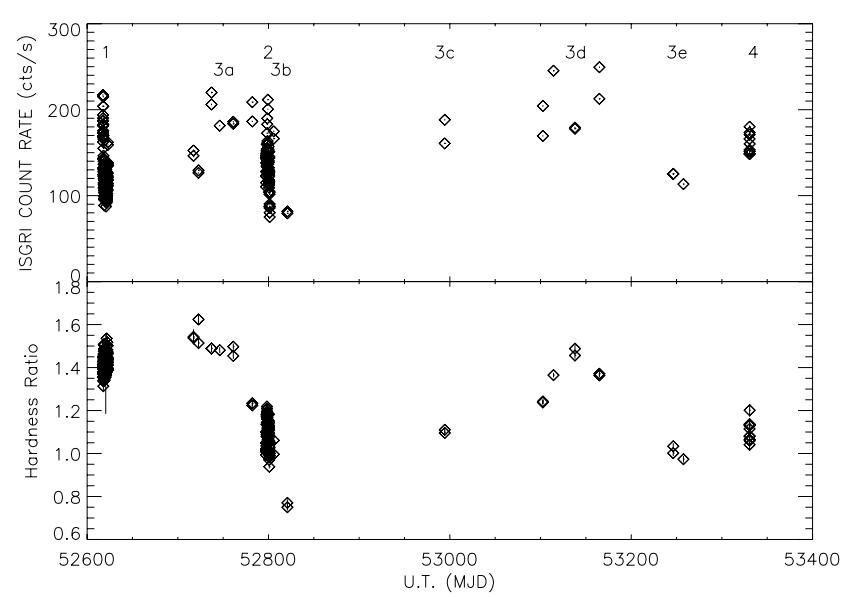

Fig. 2. The 20-200 keV IBIS/ISGRI light curve of Cygnus X-1 from 2002 November 25 until 2004 November 22 and corresponding HR between the 40-100 and the $20-30 \mathrm{keV}$ energy bands (see text and Tables 1 and 2 for epoch definitions).

versions 17 and 4 respectively were taken for epoch 4, e.g., after the failure of two detectors.

\section{Results of the analysis}

As shown in Fig. 1, during the epoch 2 INTEGRAL observations, the 1.5-12 keV ASM average count rate of Cygnus X-1 ( 1.3 Crab) was larger than during epoch 1 ( $290 \mathrm{mCrab})$ by a factor of 4.5 . The derived IBIS/ISGRI $20-200 \mathrm{keV}$ light curves and Hardness Ratio (HR) of Cygnus X-1 are shown respectively in Fig. 2 (general overview of part of PV-Phase and epochs 1 to 4) and Fig. 3 (specific zooms on part of PV-Phase, epochs 1 and 2). From epoch 1 to epoch 2, while the ASM average count rate increased, the $20-200 \mathrm{keV}$ IBIS/ISGRI one decreased from $\sim 910$ to $\sim 670 \mathrm{mCrab}$ as shown in Fig. 3 (where, in the $20-200 \mathrm{keV}$ range, $1 \mathrm{Crab}=205 \mathrm{cts} \mathrm{s}^{-1}$ ). This probably indicates a state transition between epochs 1 and 2, as also suggested by the decrease in the IBIS HR (Fig. 3). Similar transition, with a change in the ASM light curves and an evolving IBIS HR, occurred again during GPS data (epoch 3). Figure 2 (bottom) shows the IBIS HR behaviour over the whole 2002-2004 period indicated in Table 1. 

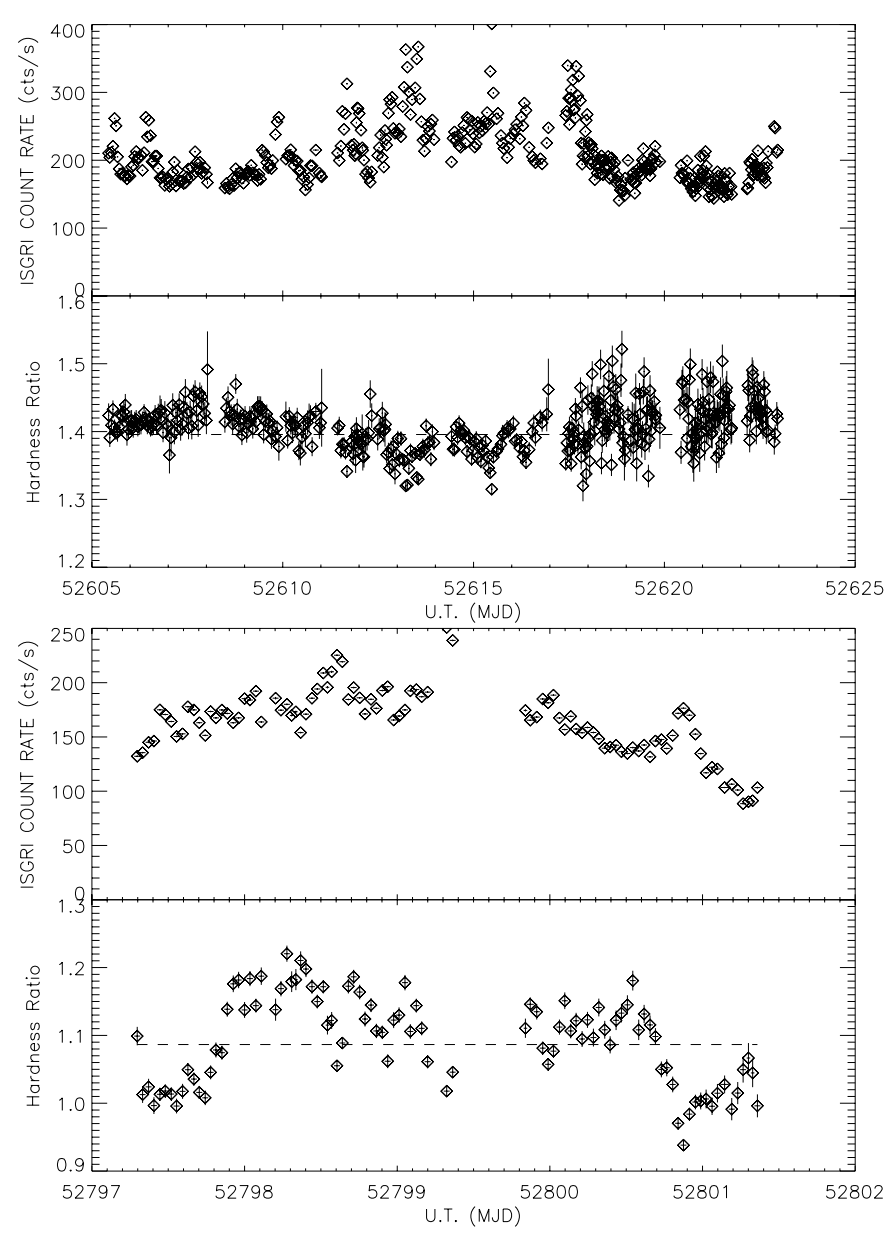

Fig. 3. Top: zoom on the 20-200 keV IBIS/ISGRI light curve of Cygnus X-1 from 2002 November 25 until December 15 and corresponding HR between the 40-100 and the 20-30 keV energy bands (average level denoted by dashed line). Bottom: same as above for epoch 2.

We sampled epoch 3 in five distinct sub-groups (noted $a$ to $e$ ) of close pointings which appear to occur, according to Figs. 1 and 2, in different regimes of ASM count rate and of average IBIS HR. The data of each epoch (and sub-group) were summed to obtain an average spectrum studied separately. We added $3 \%$ systematic errors for JEM-X (5-30 keV range), IBIS (20 keV-1 MeV range) and SPI (22 keV-1 MeV range) and fitted the resultant spectra simultaneously using XSPEC v11.3.0 (Arnaud et al. 1996). In order to account for uncertainties in the cross-calibration of each instrument, a multiplicative constant was added in the spectral fits to each instrument data set: it was set free for IBIS and SPI and frozen to 1 for JEM-X.

Several models were used in the course of the spectral analysis. In XSPEC notation, we used a multicolour disc black body DISKBB (Mitsuda et al. 1984) plus a Comptonization model COMPTT (Titarchuk 1994) and, when necessary, we added a Gaussian for the Fe line with the GAUSSIAN model and the reflection component REFLECT (Magdziarz \& Zdziarski 1995). This latter component models the X-ray reflection of the comptonized radiation from neutral or partially ionized matter, presumably the optically thick accretion disc (Done et al. 1992;
Gierliński et al. 1997, 1999; Magdziarz \& Zdziarski 1995). For this source, we always used a fixed absorption column density $N_{\mathrm{H}}$ of $6 \times 10^{21} \mathrm{~cm}^{-2}$ (Balucińska-Church et al. 1995). We also tied the input soft photon temperature $k T_{0}$ of the COMPTT model to the inner disc temperature $k T_{\text {in }}$ value found by the DISKBB model. In order to compare all our data (from epochs 1 to 4) with the same model, we show the parameters obtained from the current fitted model described above (multicolour disc black body plus Comptonization convolved by reflection and Gaussian when necessary). We also tried more complex models such as COMPPS and EQPAIR, developed respectively by Poutanen \& Svensson (1996) and Coppi (1999), coupled to the GAUSSIAN and the DISKBB (or DISKPN for EQPAIR only, see Sect. 3.2) models: we present such results only for epoch 2, when the statistics were significantly better, the instrument configurations stable and during which the presence of a nonthermal component appeared more pronounced.

\subsection{The hard state spectrum}

Figure 4 shows the resultant count spectrum obtained in epoch 1 (2002 December 9-11) from $5 \mathrm{keV}$ up to $1 \mathrm{MeV}$ with the JEM-X, SPI, IBIS/ISGRI and PICsIT data. A simple power law model does not fit the spectra well (photon index $\Gamma$ of $1.9 \pm 0.1$ and reduced chi-square $\chi_{\text {red }}^{2}=12.90$ with 213 degrees of freedom, hereafter d.o.f.). A cutoff in the model, with a typical folding energy of approximately $150 \mathrm{keV}$, clearly improves the fit $\left(\chi_{\text {red }}^{2}=2.12\right.$ with 212 d.o.f.) and better describes the available data. Since a cutoff power law is usually attributed to thermal Comptonization, we replaced this phenomenological model by a more physical model of Comptonization (COMPTT, Titarchuk 1994). Some residuals were still visible around $10 \mathrm{keV}$ so we added a model of reflection (with an inclination angle equal to $45^{\circ}$ ) to account for this excess. The final tested model therefore includes thermal Comptonization convolved by reflection (REFLECT), with solar abundances for Fe and He (Anders \& Grevesse 1989). We obtain a plasma temperature $k T_{\mathrm{e}}$ of $67 \mathrm{keV}$ with an optical depth $\tau$ of 1.98 and $\Omega / 2 \pi=0.25$, with $\chi_{\text {red }}^{2}=1.45$ (230 d.o.f.). The disc black body is very weak or below the energy range of JEM-X: this component was not used in our fits. As it gives no contribution, we froze the $k T_{0}$ temperature of COMPTT at $0.20 \mathrm{keV}$. Normalization constants between instruments (JEM$\mathrm{X}, \mathrm{IBIS} / \mathrm{ISGRI}$, SPI, IBIS/PICsIT) are respectively equal to 1 , 1.2, 1.3 and 0.9 .

Note that some residual points are visible in this plot, in particular for the SPI data at low energies (and for the IBIS data around $200 \mathrm{keV}$ ). This is mostly related to the non perfect cross-calibration between the INTEGRAL instruments. Indeed, by fitting separately the different instrument data, the residuals are reduced, with little change in the spectral parameters. Future improvement in the cross-calibration of INTEGRAL telescopes will allow a better determination in the relative flux normalizations and also a better agreement of the derived spectral shapes. The IBIS configuration was not stable in the first phases of the mission: in particular, the PV-Phase spectra may suffer from the fact that the IBIS responses were built from the 
Table 2. Best-fit parameters of Cygnus X-1 for the current thermal model in the different observation epochs.

\begin{tabular}{ccclccccc}
\hline Epoch & $\begin{array}{c}\text { Dates } \\
(\mathrm{MJD})\end{array}$ & Disc Norm. $^{a}$ & $\begin{array}{l}k T_{\text {in }} \text { or } k T_{0} \\
(\mathrm{keV})\end{array}$ & $\begin{array}{l}k T_{\mathrm{e}} \\
(\mathrm{keV})\end{array}$ & $\tau$ & $\begin{array}{c}E_{\mathrm{Fe}} \text { line } \\
(\mathrm{keV})\end{array}$ & $\Omega / 2 \pi^{b}$ & $\begin{array}{c}\chi_{\text {red }}^{2} \\
(\mathrm{~d} . \mathrm{o.f})\end{array}$ \\
\hline 1 & $52617-52620$ & - & 0.20 (frozen) & $67_{-6}^{+8}$ & $1.98_{-0.23}^{+0.21}$ & - & $0.25_{-0.04}^{+0.03}$ & $1.45(230)$ \\
2 & $52797-52801$ & $250_{-59}^{+89}$ & $1.16 \pm 0.07$ & $100_{-17}^{+29}$ & $0.98_{-0.28}^{+0.25}$ & $7.07_{-0.11}^{+0.12}$ & $0.57_{-0.06}^{+0.09}$ & $1.69(236)$ \\
$3 a$ & $52710-52780$ & - & 0.20 (frozen) & $68_{-12}^{+22}$ & $2.08_{-0.84}^{+0.51}$ & $6.48 \pm 0.13$ & $0.32_{-0.07}^{+0.05}$ & $1.07(190)$ \\
$3 b$ & $52801-52825$ & $312_{-24}^{+25}$ & $1.15 \pm 0.03$ & $93 \pm 42$ & $0.80_{-0.40}^{+0.86}$ & $6.40 \pm 0.73$ & $0.58_{-0.18}^{+0.20}$ & $0.93(190)$ \\
$3 c$ & 52990 & $361_{-67}^{+61}$ & $0.99 \pm 0.08$ & $58_{-15}^{+54}$ & $1.60_{-0.80}^{+0.64}$ & $6.96 \pm 0.19$ & $0.23_{-0.09}^{+0.17}$ & $0.99(190)$ \\
$3 d$ & $53101-53165$ & - & 0.20 (frozen) & $56_{-7}^{+12}$ & $2.28_{-0.41}^{+0.30}$ & $6.11 \pm 0.26$ & $0.27 \pm 0.06$ & $0.81(190)$ \\
$3 e$ & $53240-53260$ & $132 \pm 10$ & $1.39 \pm 0.77$ & $48_{-6}^{+20}$ & $1.85_{-0.07}^{+0.40}$ & $6.49 \pm 0.38$ & $0.49_{-0.32}^{+0.37}$ & $1.56(190)$ \\
4 & 53335 & $232_{-32}^{+21}$ & 1.16 (frozen) & $128_{-63}^{+84}$ & $0.74_{-0.38}^{+0.88}$ & $7.78_{-0.42}^{+0.44}$ & $0.47_{-0.14}^{+0.18}$ & $0.97(221)$ \\
\hline
\end{tabular}

Notes:

${ }^{a}$ Disc normalization $\mathrm{K}$ is given by $K=(R / D)^{2} \cos \theta$ where $R$ is the inner disc radius in units of $\mathrm{km}, D$ is the distance to the source in units of $10 \mathrm{kpc}$ and $\theta$ the inclination angle of the disc.

${ }^{b}$ Solid angle of the reflection component.

Model applied in XSPEC notations: CONSTANT*WABS*(DISKBB+GAUSSIAN+REFLECT*COMPTT) with $N_{\mathrm{H}}$ fixed to $6 \times 10^{21} \mathrm{~cm}^{-2}$ and $\mathrm{kT}_{0}$ value tied to disc $k T_{\text {in }}$. Errors are at $90 \%$ confidence level $\left(\Delta \chi^{2}=2.7\right)$.

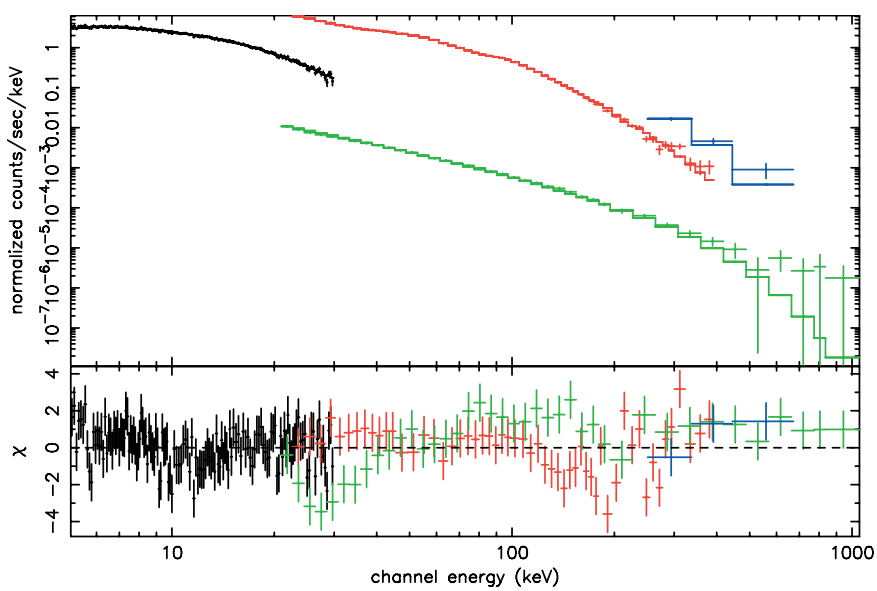

Fig. 4. Spectra of Cygnus X-1 in 2002 December 9-11 (epoch 1) with the JEM-X (black), SPI (green) and IBIS (ISGRI: red; PICsIT: blue) data. The best-fit model is a Comptonization model with reflection (see Table 2). Residuals in $\sigma$ units are also shown.

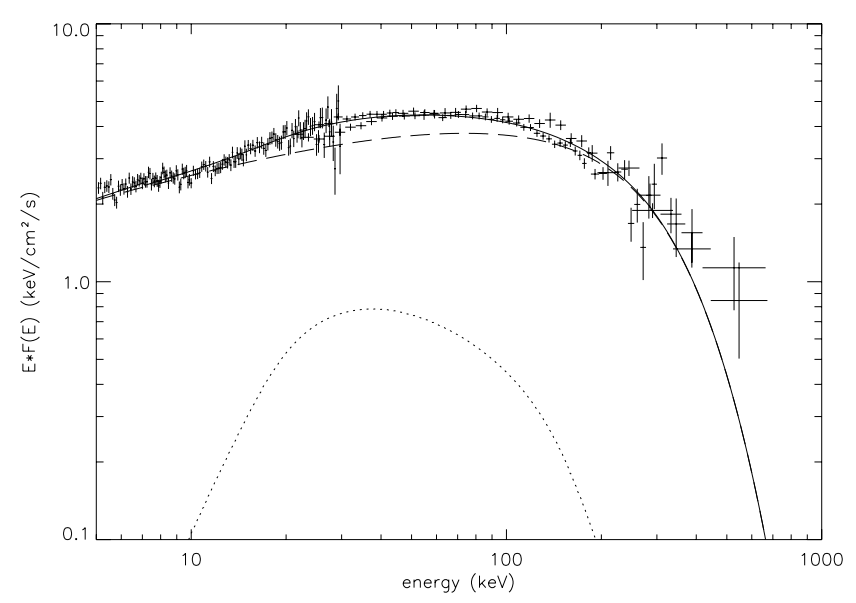

Fig. 5. Epoch 1 unabsorbed $E F(E)$ spectrum of Cygnus X-1 along with the best-fit model described in Table 2 with the JEM-X, SPI and IBIS (ISGRI and PICsIT) data. Dotted: reflection. Long dashes: comptonization. Thick: total model.

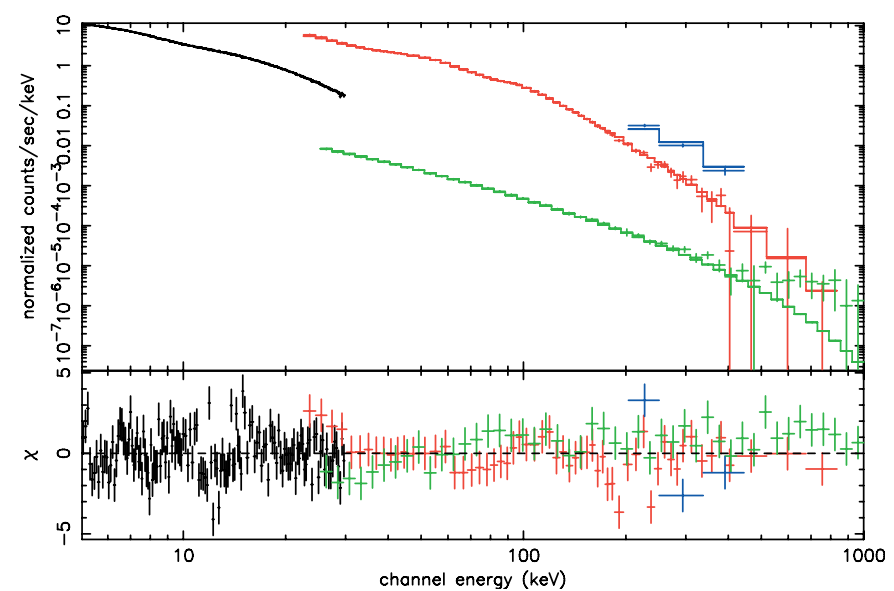

Fig. 6. Spectra of Cygnus X-1 in 2003 June 7-11 (epoch 2) with the JEM-X (black), SPI (green) and IBIS (ISGRI: red; PICsIT: blue) data. The best-fit model is a multicolour disc black body and a Comptonization model with Gaussian and reflection components (see Table 2). Residuals in $\sigma$ units are also shown.

Crab nebula observations performed in later periods, with a defined and stable configuration.

In spite of these caveats, all spectra overlap relatively well and do define the same set of spectral parameters. Figure 5 shows the resultant $E F(E)$ spectrum and its best-fit with the JEM-X, IBIS and SPI data. Table 2 summarizes all fit results. While the $20-100 \mathrm{keV}$ luminosity is $6.5 \times 10^{36} \mathrm{erg} \mathrm{s}^{-1}$ (with a distance to the source fixed at $2.4 \mathrm{kpc}$ ), the bolometric luminosity (extrapolated from $0.01 \mathrm{keV}$ to $10 \mathrm{MeV}$ ) has the value of $2.2 \times 10^{37} \mathrm{erg} \mathrm{s}^{-1}$. The best-fit parameters we obtain are consistent with those found in $\mathrm{BH}$ binaries in the HS (McClintock \& Remillard 2003) and with previous results reported on Cygnus X-1 in the HS, in particular from the INTEGRAL data (Bazzano et al. 2003; Bouchet et al. 2003; Pottschmidt et al. 2003b). These authors generally give plasma temperatures between $50-100 \mathrm{keV}$ and optical depths in the 1.0-2 range. In particular, our SPI spectrum is fully compatible with the one reported by Bouchet et al. (2003). These authors observed that the derived spectrum had an excess component 
with respect to a Sunyaev \& Titarchuk (S-T) Comptonization model (1980) and that it was better described by a cutoff power law with $\Gamma=1.5$ and a cutoff energy of $155 \mathrm{keV}$. We find exactly the same results for our SPI spectrum alone if we fit it with an S-T model or a cutoff power law. This excess emission, with respect to the simple S-T model, was already observed in a number of cases for the HS spectra of BH systems, e.g., Jourdain \& Roques (1994). However, with the Titarchuk (1994) model (which better describes the Comptonization for high plasma temperatures), no significant excess is observed in epoch 1 spectra of Cygnus X-1.

\subsection{The transition to a softer state in 2003 June}

As visible in the change of the ASM light curve of Cygnus X-1 (see Fig. 1) and its corresponding high-energy HR shown in Fig. 3 (average value of 1.1 for epoch 2 compared to more than 1.4 for epoch 1 ), the source softens during epoch 2. Although a single power law does not fit the data properly $\left(\chi_{\text {red }}^{2}=23.51\right.$ with 245 d.o.f.), the derived slope is softer $(\Gamma=2.2 \pm 0.1)$. As for the HS spectrum analysis, an inspection of the residuals reveals what kind of component can be added to build a proper model. The need for each new component is then checked with the results of the fits and the final model is further verified by repeating the procedure with components added in a different order.

First, we included a cutoff in the power law and we improved the $\chi^{2}$ by $\Delta \chi^{2}=7.8$ : this indicates that the new component is significant at more than the $95 \%$ confidence level. A cutoff in the power law model, with a typical folding energy of approximately $200 \mathrm{keV}$, better describes the available data, so we tried the physical Comptonization model (COMPTT) as we did for epoch 1 . Moreover, since very large residuals are visible in the soft X-rays and since we suspect a transition to the IS, we added a multicolour disc black body (DISKBB), which is in fact required by the data $\left(\chi_{\text {red }}^{2}=14.87\right.$ and 239 d.o.f. without this component). Some residuals around $10 \mathrm{keV}$ indicate the need for a reflection component. Therefore, the best model for the continuum consists of a multicolour disc black body (DISKBB) and thermal Comptonization (COMPTT) convolved by reflection (REFLECT, with parameters as above). The Comptonization temperature $k T_{0}$ was fixed to the $k T_{\text {in }}$ value returned by the DISKBB. Finally, we added a Gaussian to account for the residuals in the JEM-X data around 6-7 keV $\left(\chi_{\text {red }}^{2}=2.42\right.$ and 237 d.o.f. without this component).

Table 2 summarizes the best-fit parameters and the $\chi_{\text {red }}^{2}$ obtained from $5 \mathrm{keV}$ up to $1 \mathrm{MeV}$. We obtained a plasma temperature $k T_{\mathrm{e}}$ of $100_{-17}^{+29} \mathrm{keV}$ and an optical depth $\tau$ of $0.98_{-0.28}^{+0.25}$, respectively higher and lower than in epoch $1\left(k T_{\mathrm{e}}=67_{-6}^{+8} \mathrm{keV}\right.$, $\left.\tau=1.98_{-0.23}^{+0.21}\right)$. The inner disc temperature reached $1.16 \mathrm{keV}$ and a significant line is detected at a centroid energy of $7.07 \mathrm{keV}$, with an Equivalent Width $(E W)$ of $1.4 \mathrm{keV}$. With the same assumed distance, the luminosity is $6.5 \times 10^{36} \mathrm{erg} \mathrm{s}^{-1}$ in the $0.5-10 \mathrm{keV}$ range and $5.2 \times 10^{36} \mathrm{erg} \mathrm{s}^{-1}$ in the $20-100 \mathrm{keV}$ band. The bolometric luminosity, extrapolated from $0.01 \mathrm{keV}$ up to $10 \mathrm{MeV}$, has the value of $2.0 \times 10^{37} \mathrm{erg} \mathrm{s}^{-1}$; the disc accounts for $26 \%$ of the total luminosity.
The derived disc normalization is possibly underestimated and not well constrained by the JEM-X data (which start at $5 \mathrm{keV}$ ). The multicolour disc black body is only an approximation of the soft component: the direct derivation of physical parameters of the disc from the best-fit values may suffer from some important effects (Merloni et al. 2000). According to these authors, the dominant effect seems to be that, in the inner part of the disc, the opacity is dominated by electron scattering rather than free-free absorption. The net result is that the derived temperature given by the $k T_{\text {in }}$ parameter overestimates the effective inner temperature by a factor of 1.7 or more (Shimura \& Takahara 1995). This has an effect on the estimation of the inner disc radius as well. Assuming an inclination angle of $45^{\circ}$, the derived internal radius from the best-fit disc normalization has the unphysical value of $4.50_{-0.56}^{+0.74} \mathrm{~km}$. For a $10 M_{\odot} \mathrm{BH}$, this value corresponds to $0.15 R_{\mathrm{S}}$ (where $R_{\mathrm{S}}$ is the Schwarzschild radius), i.e., smaller than the last stable orbit even around a maximally spinning Kerr BH.

A comparatively strong reflection component $(\Omega / 2 \pi=$ $0.57_{-0.06}^{+0.09}$ while it was $0.25_{-0.04}^{+0.03}$ for epoch 1$)$ is also necessary to fit the data $\left(\chi_{\text {red }}^{2}=4.57\right.$ and 237 d.o.f. without this component). Our $\Omega / 2 \pi$ value is consistent with $A S C A$, RXTE (Gierliński et al. 1996, 1999) and BeppoSAX observations (Frontera et al. 2001). The best-fit model, over-plotted on data from JEM-X, SPI, IBIS/ISGRI and PICsIT, is reported in count units in Fig. 6. Figure 7 shows the resultant $E F(E)$ spectrum with its best-fit. Note that we have rebinned, for illustrative purposes, the SPI high-energy points above $750 \mathrm{keV}$ to reach the level of $3 \sigma$, but the fit was performed on the original energy channels of the spectrum (50 bins over the $22 \mathrm{keV}-1 \mathrm{MeV}$ band). Normalization constants are respectively equal to $1,1,1.2$ and 1 . Residuals again show that improvements in the instrument cross-calibration and responses are still needed to obtain a fully satisfactory fit of the INTEGRAL combined data, but we believe that the general spectral model and parameters are well determined.

Considering the behaviour of the ASM light curve (Fig. 1), the evolution of the high-energy IBIS HR (Fig. 3, lower panel), the relative softness of the spectrum and the presence of a relatively strong hard energy emission, it appears that during the 2003 June observations Cygnus X-1 was in the IS (or in the FST). This is also confirmed by radio observations of Malzac et al. (2004) who suggested that the fluctuations of the radio luminosity were associated with a pivoting of the highenergy spectrum. The derived thermal Comptonization parameters are consistent with those found in $\mathrm{BH}$ binaries in soft states (McClintock \& Remillard 2003).

As it can be seen in Fig. 7, an excess with respect to the Comptonized spectrum above $400 \mathrm{keV}$ is observed in the SPI data that is not present in epoch 1 . We tried different models of the background, we used different selections of pointings with two data processings (the standard pipeline and the specific softwares developed by the SPI instrument team); however, this feature is always present. To evaluate the possible presence of an instrumental feature, we re-analyzed the Crab observation performed with SPI on 2003 February 22-24, with the same configuration and dither pattern as those of our Cygnus X-1 observations. We obtained a spectrum consistent with the one 


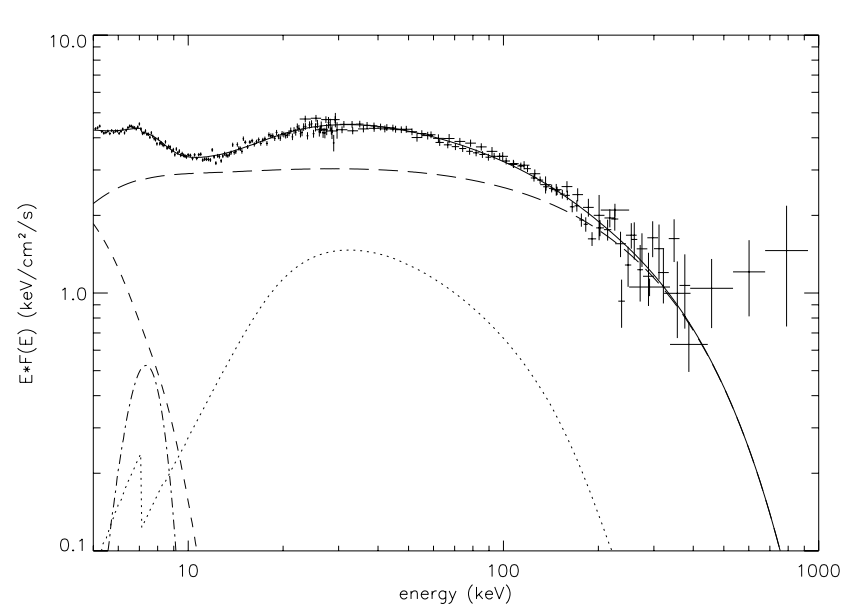

Fig. 7. Epoch 2 unabsorbed $E F(E)$ spectrum of Cygnus X-1 along with the best-fit model described in Table 2 with the JEM-X, SPI and IBIS (ISGRI and PICsIT) data. Dashed: Disc. Dotted-dashed: Gaussian line. Dotted: Reflection. Long dashes: Comptonization. Thick: Total model.

reported by Roques et al. (2003). With 3\% systematics added to the $200 \mathrm{ks}$ SPI data, the Crab spectrum is well described by a power law with $\Gamma=2.1$ over the $22-1000 \mathrm{keV}$ energy range and no high-energy excess above $400 \mathrm{keV}$ is observed. This test shows that it is unlikely that the observed high-energy tail in the Cygnus X-1 IS spectrum is due to a systematic effect.

We fitted this excess with an extra component, in addition to our current Comptonization model. Using a power law for all the data (except JEM-X), we obtain a best-fit photon index $\Gamma$ of $2.12_{-0.16}^{+0.31}$. The improvement in the $\chi_{\text {red }}^{2}$ is relatively important (1.65 with an absolute chi-square reduced by 10 for 2 additional free parameters), making this component significant. An F-test (Bevington \& Robinson 1992) provides a chance probability that this improvement is due to fluctuations of about $10^{-2}$ (but see Protassov et al. 2002 about the limitations of the Ftest). At the same time, the parameters obtained for the current model are different to those obtained without the power law. We obtained a plasma temperature of $50 \mathrm{keV}$, an optical depth of 2.1 and $\Omega / 2 \pi$ is around 0.49 . While the IBIS high-energy data do not require this additional component, IBIS points are also consistent, due to the large error bars, with this additional power law model. A better $\chi_{\text {red }}^{2}$ (equal to 1.55 , with an absolute chi-square reduced by 35 , giving now a chance probability of $10^{-5}$ for the additional power law that the improvement is due to fluctuations) is obtained when PICsIT data are neglected. Since the SPI telescope is certainly the best calibrated INTEGRAL instrument at energies higher than $400-500 \mathrm{keV}$, we consider this feature to be significant.

Consequently, in order to account for this high-energy emission with more physical models, we fitted the data with the hybrid models COMPPS and EQPAIR (coupled to the usual disc and Gaussian line components). They combine both thermal and non-thermal particle distributions in the calculation of the emergent spectrum, as fully described by Poutanen \& Svensson (1996) and Coppi (1999). Those models use a hot plasma cloud, mainly modeled as a spherical corona around the compact object (configuration taken in our fits) or as the well-known "slab" geometry, illuminated by soft thermal photons coming from an accretion disc. These photons are Compton scattered by both thermal (Maxwellian) and nonthermal (power law) electrons that lose energy by Compton, Coulomb and bremsstrahlung interactions. The number of electrons is determined by $\tau$, the corresponding (total) vertical Thomson optical depth of the corona.

Both EQPAIR and COMPPS models allow one to inject a nonthermal electron distribution with Lorentz factors between $\gamma_{\text {min }}$ and $\gamma_{\max }$ and a power law spectral index $\Gamma_{\mathrm{p}}$. In the EQPAIR model, the system is characterized by the power $L_{\mathrm{i}}$ supplied to its different components, expressed as the dimensionless compactnesses $l_{\mathrm{i}}=\left(L_{\mathrm{i}} \sigma_{\mathrm{T}}\right) / R m_{\mathrm{e}} c^{3}$ (where $R$ is the corona size). This model has two advantages with respect to the COMPPS: first, it is valid both for high $k T_{\mathrm{e}}$ and low $\tau$ and vice-versa (while COMPPS should be used for $\tau<3$ only). Secondly, the EQPAIR model also takes into account the electron-positron annihilation process. We used the DISKPN and DIKSBB routines in the XSPEC package to describe the soft emission from the accretion disc in the two hybrid models (EQPAIR and then COMPPS). With the DISKPN, the main characteristic is that the disc spectrum incident on the plasma is computed assuming a pseudoNewtonian potential for the accretion disc, extending from 3 (the minimum stable orbit) to $500 R_{\mathrm{s}}$.

Both the purely thermal and the thermal/non-thermal bestfit parameters of these hybrid models for the Cygnus X-1 spectrum of 2003 June are reported in Table 3. The pure thermal models obviously do not improve the fit with respect to the thermal Comptonization model, but when the effect of a nonthermal electron distribution is included, the $\chi^{2}$ are reduced by a significant amount and the high-energy component appears well fitted by these models, in particular by the non-thermal EQPAIR.

First, for EQPAIR, with frozen values of $\gamma_{\min }$ and $\gamma_{\max }$ at respectively 1.5 and 1000 and with the same value for $N_{\mathrm{H}}$ and abundances as before, we obtain the dimensionless parameters $l_{\mathrm{h}} / l_{\mathrm{s}}=4.57_{-0.87}^{+0.04}$ and $l_{\mathrm{nth}} / l_{\mathrm{h}}=0.16_{-0.08}^{+0.11}$ (where $l_{\mathrm{s}}, l_{\text {th }}, l_{\mathrm{nth}}$ and $l_{\mathrm{h}}=l_{\text {th }}+l_{\text {nth }}$ correspond to the compactness in soft disc photons irradiating the plasma, in direct thermal electron heating, in electron acceleration and in total power supplied to electrons in the plasma respectively). These parameters indicate that the power in the non-thermal component is $\sim 16 \%$ of the total power supplied to the electrons in the corona. With a $\chi_{\text {red }}^{2}=1.55$ (232 d.o.f.), clearly better than the current epoch 2 model and the pure thermal version of the EQPAIR model (values also reported in Table 3), the derived thermal values of $\tau, \Omega / 2 \pi, E_{\mathrm{Fe}}$ centroid and EW match, within the uncertainties, the parameters obtained in Table 2. The value of $k T_{\mathrm{e}}$ decreases from the pure thermal model as expected and it is similar to the value we get when adding a power law to our current model. While fitting a soft state spectra from $0.5-200 \mathrm{keV}$ with the same hybrid model, Frontera et al. (2001) found $\Gamma_{\mathrm{p}}$ equal to $2.5 \pm 0.1$, an $E W \sim 300 \mathrm{eV}$ and $\Omega / 2 \pi$ around 0.63 . These values match well with our non-thermal results. However, they obtained a lower $l_{\mathrm{h}} / l_{\mathrm{s}} \sim 0.36$ and a higher $l_{\mathrm{nth}} / l_{\mathrm{h}} \sim 0.77$. For what they called a typical soft state, McConnell et al. (2002) found a $\Gamma_{\mathrm{p}}$ 
Table 3. Best-fit parameters of Cygnus X-1 for thermal/non-thermal hybrid models in the epoch 2 observations.

\begin{tabular}{ccccc}
\hline Model & eqpair (thermal) & eqpair (non-thermal) & compps (thermal) & compps (non-thermal) \\
\hline$l_{\mathrm{h}} / l_{\mathrm{s}}$ & $5.3_{-0.4}^{+0.8}$ & $4.57_{-0.87}^{+0.04}$ & - & - \\
$l_{\text {nth }} / l_{\mathrm{h}}$ & - & $0.16_{-0.08}^{+0.11}$ & - & - \\
$\tau$ & $0.66_{-0.09}^{+0.02}$ & $0.49_{-0.02}^{+0.24}$ & $0.57_{-0.01}^{+0.03}$ & $1.43 \pm 0.10$ \\
$\Gamma_{\mathrm{p}}$ & - & $2.4_{-1.0}^{+0.5}$ & - & $3.72_{-0.22}^{+0.017}$ \\
$\gamma_{\min }, \gamma_{\max }$ & - & $1.5,1000$ (frozen) & - & $1.34,1000$ (frozen) \\
$\Omega / 2 \pi$ & $0.73_{-0.03}^{+0.07}$ & $0.63 \pm 0.08$ & $0.60 \pm 0.05$ & $0.87_{-0.28}^{+0.12}$ \\
$E_{\mathrm{Fe}}(\mathrm{keV})$ & $7.18_{-0.14}^{+0.16}$ & $7.06 \pm 0.06$ & $7.20_{-0.22}^{+0.10}$ & $7.14 \pm 0.09$ \\
$E W(\mathrm{eV})$ & 287 & 379 & 238 & 412 \\
$k T_{0}(\mathrm{keV})$ & $1.44_{-0.03}^{+0.07}$ & $1.39_{-0.01}^{+0.27}$ & 1.20 (frozen) & 1.20 (frozen) \\
$k T_{\mathrm{e}}(\mathrm{keV})$ & $103^{a}$ & $42^{a}$ & $111_{-6}^{+3}$ & $39_{-2}^{+33}$ \\
$\chi_{\text {red }}^{2}($ d.o.f.) & $1.68(234)$ & $1.55(232)$ & $1.72(233)$ & $1.69(230)$ \\
\hline
\end{tabular}

Notes:

${ }^{a}$ The electron temperature is calculated for the best-fit model (i.e., not free parameters).

Model applied in XSPEC notations: CONSTANT*WABS*(DISKPN+GAUSSIAN+EQPAIR) or CONSTANT*WABS*(DISKBB+GAUSSIAN+COMPPS) with $N_{\mathrm{H}}$ fixed to $6 \times 10^{21} \mathrm{~cm}^{-2}$ and $k T_{0}$ value tied to disc $k T_{\text {in }}$. Errors are at $90 \%$ confidence level $\left(\Delta \chi^{2}=2.7\right)$.

compatible with ours (around 2.6) but, again, $l_{\mathrm{h}} / l_{\mathrm{s}} \sim 0.17$ and $l_{\text {nth }} / l_{\mathrm{h}} \sim 0.68$ different from our values. For another soft state, Gierliński et al. (1999) found even higher values for these latter three parameters, in the range 2.6-3.4, 0.25-1.6 and 0.77-1. All these comparisons suggest that, in epoch 2, Cygnus X-1 was not in the typical soft state, but was rather in the IS.

Second, the thermal part of the hybrid model compPs fits the data for a $\chi_{\text {red }}^{2}=1.72$ (233 d.o.f.). Most of the crucial parameters like $k T_{\mathrm{e}}$, the optical depth, $\Omega / 2 \pi$ and the $E_{\mathrm{Fe}}$ centroid are compatible with those obtained with our current model (reported in Table 2) with a comparable $\chi_{\text {red }}^{2}$. Trying to improve our fits, we then used the non-thermal part of the coMPPS model with an electron distribution of index $3.72_{-0.22}^{+0.17}$ and we report all the derived parameters in Table 3. Comparing the different $\chi_{\text {red }}^{2}$ (now equal to 1.69 for 230 d.o.f.), the improvement is significant but not as strong as the EQPAIR non-thermal model. Again, $k T_{\mathrm{e}}$ decreases from the value obtained with a pure thermal model, which is compatible with McConnell et al. (2002). Using the non-thermal part of the COMPPS model to fit a soft state, these authors found temperatures $(\sim 60 \mathrm{keV})$, reflection factors and energy index $\Gamma_{\mathrm{p}}(\sim 3.5)$ again compatible with ours.

Some authors, e.g., Belloni et al. (1996) and Ibragimov et al. (2005, submitted) claim that the soft states observed in 1996, 1998 and 1999 were in fact IS. Modeling the spectra with EQPAIR and a non-thermal high-energy component, Ibragimov et al. showed spectral results consistent with the non-thermal values we report here, including a high $l_{\mathrm{h}} / l_{\mathrm{s}}$ value. According to the different soft states of Cygnus X-1 analyzed, they obtained for this parameter values between 4.2 and 12 and for $\tau$, values in the range $0.9-1.6$. These results are consistent with ours.

We find that the non-thermal hybrid EQPAIR model the best able to fit our epoch 2 data: this model clearly accounts for the high-energy tail observed. The non-thermal component represents $16 \%$ of the total power supplied to the electrons in the corona and the inferred luminosity in the $20-100 \mathrm{keV}$ range is $6 \times 10^{36} \mathrm{erg} \mathrm{s}^{-1}$ while the bolometric one is $3.3 \times 10^{37} \mathrm{erg} \mathrm{s}^{-1}$.

\subsection{Evolutions occurring during epochs 3 and 4 (2003-2004)}

We separated epoch 3 in five sub-groups as follows: $a$ ) before epoch 2 (MJD 52710-52780); b) just after epoch 2 (MJD 52801-52825); c) at MJD = 52990 when the source switches again to a softer state; $d$ ) when a harder state is then observed (MJD 53101-53165); e) observations when the ASM count rate slightly increases while the IBIS HR decreases (MJD 53240-53260). The IBIS HR shown in Fig. 2 indicates spectral evolution with time: from groups $a$ to $e$, the mean HR value is $1.55,0.8,1.1,1.4$ and 1.05 . Accordingly, a simple power law model fits the data with different indexes: $\Gamma=1.9$, 2.3, 2.2, 1.9 and $2.3 \pm 0.1$ for groups $a$ to $e$.

We fitted from $5 \mathrm{keV}$ to $400 \mathrm{keV}$ the JEM-X and IBIS/ISGRI spectra of the data collected during these subgroups, using our current Comptonization (COMPTT) model for the HS and the IS defined above. We fixed the seed photon temperature $k T_{0}$ to the $k T_{\text {in }}$ value given by the DISKBB (or frozen to $0.20 \mathrm{keV}$ when the disc emission is not detected). The best-fit parameters are listed in Table 2. Normalization constants between the two instruments are very close to each other ( 1-1.2 for IBIS/ISGRI when JEM-X constant is frozen to 1). Variations in amplitude and overall spectral shape are observed between the epoch 3 sub-groups. While no disc emission is detected for groups $a$ and $d$, a disc component is required for groups $b, c$ and $e$. The $E W \mathrm{~s}$ are also changing: the softer the source, the larger the $E W(\sim 110 \mathrm{eV}$ for group $a$ compared to $\sim 780 \mathrm{eV}$ for group $e$ ). These results and the IBIS HR (Fig. 2) indicate that, during sub-groups $3 a$ and $d$, Cygnus $\mathrm{X}-1$ was in a HS (as in epoch 1) while, in sub-groups $3 b, c$ and $e$, the source was in a softer state.

During epoch 4, the simple power law slope is $\Gamma=2.2 \pm 0.1$ and the mean IBIS HR value is 1.1. This indicates that the 


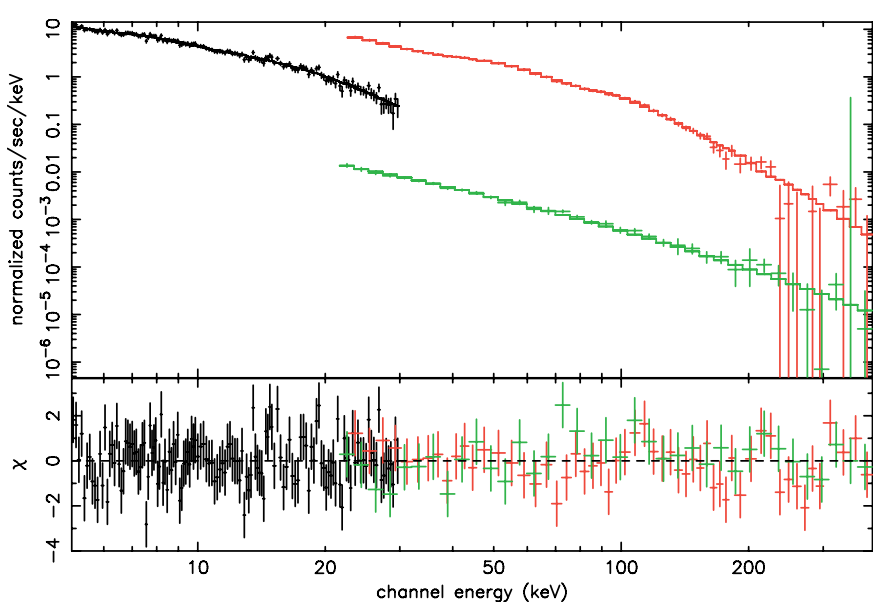

Fig. 8. Epoch 4 spectra of Cygnus X-1 with the JEM-X (black), SPI (green) and IBIS/ISGRI (red) data along with the best-fit current model (see Table 2). Residuals in $\sigma$ units are also shown.

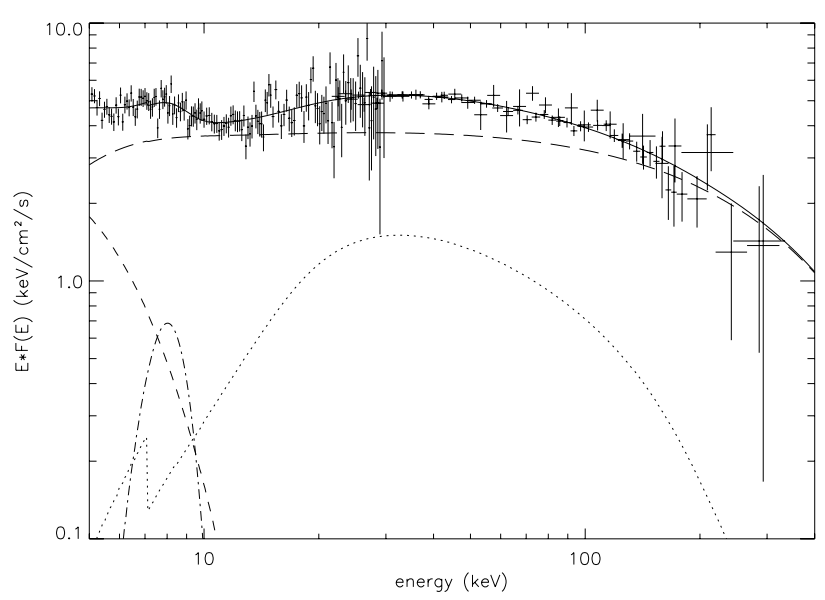

Fig. 9. Epoch 4 unabsorbed $E F(E)$ spectrum of Cygnus X-1 along with the best-fit model described in Table 2 with the JEM-X, IBIS/ISGRI and SPI data. Dashed: Disc. Dotted-dashed: Gaussian line. Dotted: Reflection. Long dashes: Comptonization. Thick: Total model.

source was again in a softer state than the HS. The count spectrum is presented in Fig. 8. Figure 9 shows the resultant $E F(E)$ spectrum and its best-fit model overplotted on the data obtained from JEM-X, IBIS/ISGRI and SPI (high-energy IBIS points are rebinned at the $3 \sigma$ level above $200 \mathrm{keV}$ for illustrative purposes). Cross-calibration constants are respectively 1 , 1 and 1.3. Using the current Comptonization (COMPTT) model defined above for epoch 2, we present in Table 2 the best-fit parameters obtained. Since we obtained a best-fit solution similar to the one of epoch 2, we fixed, to define the errors for the other parameters, the $k T_{\text {in }}$ to the epoch 2 value of $1.16 \mathrm{keV}$ and we obtained $k T_{\mathrm{e}}=128 \mathrm{keV}, \tau=0.74, \Omega / 2 \pi=0.47_{-0.14}^{+0.18}$ (close to the epoch 2 value of $0.57_{-0.06}^{+0.09}$ ) and $E_{\mathrm{Fe}}=7.78 \mathrm{keV}$. Although the temperature is little constrained and errors are large, these values indicate that the source was again in a rather soft state.

\section{Discussion}

During the broad-band ( $5 \mathrm{keV}-1 \mathrm{MeV}$ ) INTEGRAL observations of Cygnus X-1 presented in this paper, the source was detected in at least two different spectral states. For epoch 1 (part of PV-Phase) and during some of the GPS observations (epoch 3, sub-groups $a$ and $d$ ), the source was in the typical HS with a high-energy spectrum extending up to $800 \mathrm{keV}$, well characterized by a thermal Comptonization model. Parameters are typical of $\mathrm{BH}$ binaries and consistent with previous observations of Cygnus X-1 in the HS. We find that the Comptonization component must be modified by reflection. The subtended angle we derived is also compatible with values previously found for this source in the HS, either from Ginga and CGRO/OSSE $\left(k T_{\mathrm{e}} \sim 100 \mathrm{keV}, \tau \sim 1-2\right.$, and $\Omega / 2 \pi \sim 0.19-0.34$, Gierliński et al. 1997), or from BeppoSAX $\left(k T_{\mathrm{e}} \sim 60 \mathrm{keV}, \tau \sim 1-2\right.$ and $\Omega / 2 \pi=0.25$, Frontera et al. 2001). Di Salvo et al. (2001) observed even higher temperatures for the HS, from 111 to $140 \mathrm{keV}$, and $\Omega / 2 \pi \sim 0.1-0.3$, within our error bars. Also, our results are compatible with those reported by Bazzano et al. (2003), Bouchet et al. (2003) and Pottschmidt et al. (2003b) who used INTEGRAL data from other PV-Phase observations, when Cygnus X-1 was also in the HS. We did not detect any $\mathrm{Fe}$ line nor disc emission during this period. Indeed, we could determine a $90 \%$ confidence level upper limit of $94 \mathrm{eV} \mathrm{EW}$ for a narrow line $(\sigma=0.1 \mathrm{keV})$ at $6.7 \mathrm{keV}$ and of $172 \mathrm{eV}$ EW for a broad one $(\sigma=1 \mathrm{keV})$. These limits are compatible with the typical $E W \mathrm{~s}(\sim 150 \mathrm{eV})$ of broad lines observed in HS from this BH system (Frontera et al. 2001). The lack of strong Fe line and disc emission is not surprising since, in the HS, the disc does not extend close to the $\mathrm{BH}$, its inner disc temperature is low and contribution at $>5 \mathrm{keV}$ is negligible.

Cygnus X-1 underwent a clear evolution to a softer state from epoch 1 to epoch 2. We indeed observed in 2003 June INTEGRAL data hardness variations (Figs. 2 and 3 ) and photon index changes, along with the appearance of a significant soft component well fitted by a disc black body model. The changes in the high-energy component are obvious from the best-fit parameters of the current model reported in Table 2. The Comptonization parameter $y$ is characterized by both values $\left(k T_{\mathrm{e}}, \tau\right)$ where $y=\left(k T_{\mathrm{e}} / m_{\mathrm{e}} c^{2}\right) \max \left(\tau, \tau^{2}\right)$. While $y \sim 0.51_{-0.09}^{+0.10}$ for epoch 1 , its value drops to $0.19_{-0.06}^{+0.07}$ for epoch 2 .

As shown previously, these changes were correlated with evolutions of the RXTE/ASM light curve (Fig. 1) which indicate the rise of the soft disc emission. The increase in disc emission (up to about $26 \%$ of the bolometric luminosity) combined with an increase of the inner disc temperature to about $1.16 \mathrm{keV}$ suggest that the accretion disc has extended down very close to the $\mathrm{BH}$ horizon. However, the inferred inner radius $(4.5 \mathrm{~km})$ is not compatible with the size of the innermost stable circular orbit, even for a maximally spinning Kerr BH. Besides uncertainties on the inclination angle and on the disc normalization, a number of effects can lead to such unphysical estimations. As mentioned in Sect. 3.2, electron scatterings can produce a diluted black body (Shimura \& Takahara 1995) and cause the observed temperature $k T_{\text {in }}$ to be higher and the disc normalization lower. Consequently, the inner disc radii could be underestimated by a factor of 5 or more (Merloni et al. 2000). Another 
possible explanation for such a high temperature could be that the disc emission is Comptonized by a different warm corona at a temperature of a few keV, as sometimes suggested (Frontera et al. 2001; Malzac et al. 2005).

The softening of the hard component and the appearance of the disc emission were accompanied by significant increase in reflection and changes in Fe line emission. During epoch 2 data, the Fe line is needed in the spectra (while not for epoch 1) and $\Omega / 2 \pi$ increases (it is more than twice the value found for epoch 1): this again indicates that a larger disc, closer to the $\mathrm{BH}$, reflects more radiation coming from the hot plasma. Figures 5 and 7 show the resultant unabsorbed $E F(E)$ spectra for both epochs 1 and 2: we clearly see differences in amplitudes and in the relative contributions of the various components, from soft to hard X-rays/ $\gamma$-rays. Similar spectral transitions are observed during epochs 3 and $4: y=0.57_{-0.34}^{+0.27}$ for epoch 3 sub-group $a$ (while it drops to $0.14_{-0.09}^{+0.16}$ for group $b$ ), and $y=0.18_{-0.13}^{+0.24}$ for epoch 4 . The $y$ evolution, combined with the changes in the IBIS HR and in the ASM light curve, indeed shows transitions from the HS (epoch 1, epochs $3 a$ and $d$ ) to softer states (epochs 2, $3 b, c, e$ and 4).

The transition to a soft state is generally attributed to an increase in the accretion rate. In this case, the total luminosity should increase significantly. For example, the total luminosity measured by Frontera et al. (2001) in the TDS of Cygnus X-1 was about a factor of 3 higher than in the HS, with a contribution of the disc emission higher than $50 \%$ of the total luminosity. From our analysis, the spectral transition seems to occur with only a slight change in the bolometric luminosity, mainly due to the appearance of a high-energy tail which represents less than $2 \%$ of the Eddington luminosity $\left(\sim 1.5 \times 10^{39} \mathrm{erg} \mathrm{s}^{-1}\right.$ for a $\left.10 M_{\odot} \mathrm{BH}\right)$. Therefore, we can interpret the component evolution as a spectral pivoting between soft and hard $\mathrm{X}$-rays at almost constant luminosity. This has already been reported for other BH such as for the microquasar XTE J1550564 (Rodriguez et al. 2003). When the total luminosity does not change much, the spectral evolution cannot be explained simply by a large variation in the accretion rate. An additional physical parameter (for example linked to the temperature and geometry of the Comptonizing cloud or to the magnetic field) must probably vary in the system to trigger the spectral changes from the HS to a softer state at almost constant luminosity.

While our data start at $5 \mathrm{keV}$, leading to a possible underestimation of the bolometric luminosity (which in a real soft state is dominated by the soft component), the near constancy of the luminosity between epochs 1 and 2 and other facts show that the soft state we observed in this latter period is not the typical TDS observed in Cygnus X-1. Our spectral analysis and the comparisons with previous results obtained on Cygnus X-1 (see Sect. 3.2) led us to conclude that, during epoch 2 , the source was rather in the IS. The need for a cutoff in the high-energy spectrum of epoch 2 shows that the thermal Comptonization is still a dominant process for the high-energy component. The hybrid thermal/non-thermal models lead to a significant improvement in the fit of our data, compared to the thermal Comptonization models, like COMPTT, or the pure thermal versions of EQPAIR and COMPPS. However, our best-fit non-thermal parameters are sometimes intermediate between hard and soft state values (like $l_{\mathrm{h}} / l_{\mathrm{s}}, l_{\mathrm{nth}} / l_{\mathrm{h}}$ or $\Gamma_{\mathrm{p}}$ ): this again shows that Cygnus X-1 was in the IS (or in the FST). Such a conclusion was also reached by Malzac et al. (2004) on the basis of the observed correlation of the spectral hardness with the radio flux during this period.

In recent years, it has become apparent that in the $\mathrm{HS}$, the $\mathrm{BH}$ binaries become bright in radio and display clear correlations between the X-ray and radio luminosities (Corbel et al. 2003; Gallo et al. 2003) as often observed for Cygnus X-1 (Brocksopp et al. 1999; Gleissner et al. 2004b; Nowak 2005). Models where the base of a compact jet plays a major role in the physical processes of such BH systems have been proposed (Markoff et al. 2001). In such scenarios, the high-energy emission seen during the HS is interpreted as synchrotron emission from the jet that extends from radio to hard $\mathrm{X}$-rays, naturally explaining the correlations observed during the HS. Those models explain the observed (or inferred) outflows: the radio emission is proportional to the jet power which in turn is correlated with the accretion rate and with the X-ray emission (Heinz \& Sunyaev 2003; Merloni et al. 2003) as discussed for GX 339-4 (Zdziarski et al. 2004). However, detection of radio emission was reported during the TDS, for example in the BH XTE J1650-500 (Corbel et al. 2004) and the scenario increases in complexity (see Fender et al. 2005 for a complete review). More recently, Markoff et al. (2004) have proposed jet models where the synchro-self Compton or the external Comptonization radiation are the dominant processes generating X-ray spectra in BH binaries. These models seem to fit the experimental data as well as the thermal Comptonization models do.

While we did not observe any significant high-energy tail during the PV-Phase observations, it appears that, in the SPI spectra of 2003 June, when the source was in the IS, some data points above $400 \mathrm{keV}$ are not well described by the thermal Comptonization models (see Figs. 6 and 7). All the tests we have performed using different SPI background models and the Crab nebula spectrum indicate that this feature comes from the source. Similar excess over a Comptonization law has been previously observed, in particular with $C G R O / O S S E$ (2002) which detected a power law like component extending beyond $1 \mathrm{MeV}$ during a (so classified) TDS of Cygnus X-1. In our case, the SPI data of the observed IS spectrum of Cygnus X-1 imply a rather bright high-energy tail. The bolometric luminosity of the source, including this new component, is 1.65 times higher than the one obtained with the best-fit thermal current model.

This kind of steep power law, without a high-energy break (at least up to $1 \mathrm{MeV}$ ) was modeled in the past including a nonthermal component in the accretion flow, with the so-called hybrid thermal/non-thermal models from Poutanen \& Svensson (1996) and Coppi (1999). Using these same models, we obtain a better fit of our data, which indicate the necessity to include a non-thermal distribution of electrons, with a power law energy index between 2.4-3.7, depending on the model used. $\Gamma_{\mathrm{p}}$ between 2 and 3 are expected from shock acceleration models. We find that most of the crucial parameters such as $\tau, \Omega / 2 \pi, E_{\mathrm{Fe}}$ centroid, $E W$ or $k T_{\mathrm{e}}$ are compatible with previous results reported by McConnell et al. (2002), Frontera et al. (2001) and Gierliński et al. (1999). With the non-thermal 
EQPAIR model, we found an unabsorbed bolometric luminosity of $3.3 \times 10^{37} \mathrm{erg} \mathrm{s}^{-1}$ (higher than the one obtained with the current thermal model). This value is $\sim 1.9$ times lower than the one observed by McConnell et al. (2002) in the (so classified) TDS: this, along with the differences seen in the $l_{\mathrm{h}} / l_{\mathrm{s}}, l_{\mathrm{nth}} / l_{\mathrm{h}}$ and $\Gamma_{\mathrm{p}}$ values (see Sect. 3.2), definitely shows that our epoch 2 observations did not happen during a TDS but rather in the IS, with an intermediate luminosity.

Alternatively, Comptonization on a population of (thermalized) electrons with bulk motion, e.g., Titarchuk et al. (1997), Laurent \& Titarchuk (1999), is sometimes invoked to explain the power law high-energy component. This could describe the observed high-energy emission seen in epoch 2 , as it predicts $\Gamma>2$ or even softer, even if the lower energy cutoff should be around $100 \mathrm{keV}$. In addition to hybrid thermal/nonthermal models presented above, a stratified Comptonization region, providing a larger range of both electron temperatures and optical depths, could model the spectrum. Ling et al. (1997) reached the same conclusions based on Monte Carlo modeling of $C G R O / \mathrm{BATSE}$ spectra combined with (non contemporaneous) $C G R O / C O M P T E L$ data (McConnell et al. 1994). Thermal gradients are incorporated into several other models, which then lead to the generation of a high-energy tail, e.g., Skibo \& Dermer (1995), Chakrabarti \& Titarchuk (1995) and Misra \& Melia (1996).

\section{Summary and conclusions}

Using the broad-band capability of INTEGRAL, it has been possible to accumulate a large amount of simultaneous data on Cygnus $\mathrm{X}-1$ between $5 \mathrm{keV}-1 \mathrm{MeV}$ and to follow its spectral evolution from 2002 November to 2004 November. These data were helpful to characterize the evolution of the Comptonization parameters of the source correlated to the presence of a variable disc emission, indicating transitions between the HS and a softer (Intermediate) state. We also observed the presence in the SPI data of a high-energy tail during the IS (or the FST), emerging from the Comptonization component between $400 \mathrm{keV}-1 \mathrm{MeV}$ and probably associated with a nonthermal component. The extent to which the spectrum hardens at energies approaching $1 \mathrm{MeV}$ has now become an important issue for theoretical modeling of the accretion processes and radiation mechanisms in BH binaries. Data from both IBIS and SPI instruments offer the best opportunity to define more precisely the high-energy X-ray binary spectra. We hope to further investigate this using INTEGRAL data from this source and other bright $\mathrm{BH} \mathrm{X}$-ray binaries.

Acknowledgements. We thank the anonymous referee for his/her helpful comments and suggestions. MCB thanks J. Paul for a careful reading and comments on the manuscript. AAZ has been supported by KBN grants PBZ-KBN-054/P03/2001, 1P03D01827 and 4T12E04727. We thank the ESA ISOC and MOC teams for they support in scheduling and operating observations of Cygnus X-1. The present work is based on observations with INTEGRAL, an ESA project with instruments and science data center funded by ESA member states (especially the PI countries: Denmark, France, Germany, Italy, Switzerland, Spain, Czech Republic and Poland, and with the participation of Russia and the USA).

\section{References}

Anders, E., \& Grevesse, N. 1989, Geochim. Cosmochim. Acta, 53, 197

Arnaud, K. A. 1996, ASP Conf., 101, 17

Bazzano, A., Bird, A. J., Capitanio, F., et al. 2003, A\&A, 411, L389

Balucińska-Church, M., Belloni, T., Church, M. J., et al. 1995, A\&A, 302, L5

Belloni, T., Méndez, M., van der Klis, M., et al. 1996, ApJ, 472, L107

Bevington, P. R., \& Robinson, K. D. 1992, Data Reduction and Error Analysis for the Physical Sciences, 2nd Ed., (New York: McGrawHill)

Bouchet, L., Jourdain, E., Roques J.-P., et al. 2003, A\&A, 411, L377

Bowyer, S., Byram, E. T., Chubb, T. A., \& Friedman, H. 1965, Science, 147, 394

Brocksopp, C., Fender, R. P., Larionov, V., et al. 1999, MNRAS, 309, 1063

Cadolle Bel, M., Sizun, P., Rodriguez, J., et al. 2004, accepted for publication in the Proceedings of the 35th COSPAR Scientific Assembly (ASR Publication)

Chakrabarti, S. K., \& Titarchuk, L. G. 1995, ApJ, 455, 623

Coppi, P. S. 1999, 161, 375, ASP Conf. Ser., 161, 375

Corbel, S., Nowak, M. A., Fender, R. P., et al. 2003, A\&A, 400, 1007

Corbel, S., Fender, R. P., Tomsick, A. K., et al. 2004, ApJ, 617, 1272

Di Cocco, G., Caroli, E., Celesti, E., et al. 2003, A\&A, 411, L189

Di Salvo, T., Done, C., Zycki, P. T., Burderi, L., \& Robba, N. R. 2001, ApJ, 547, 1024

Done, C., Mulchaey, J. S., Mushotzky, R. F., \& Arnaud, K. A. 1992, ApJ, 395, 275

Dove, J. B., Wilms, J., Nowak, M. A., Vaughan, B. A., \& Begelman, M. C. 1998, MNRAS, 289,729

Esin, A. A., Narayan, R., Cui, W., et al. 1998, ApJ, 505, 854

Fender, R. P., Belloni, T., \& Gallo, E. 2005, to appear in From X-ray Binaries to Quasars: Black Hole Accretion on All Mass Scales, ed. T. J. Maccarone, R. P. Fender, and L. C. Ho (Dordrecht: Kluwer), [arXiv: astro-ph/0506469]

Frontera, F., Palazzi, E., Zdziarski, A. A., et al. 2001, ApJ, 546, 1027

Gallo, E., Fender, R. P., \& Pooley, G. G., 2003, MNRAS, 334, 60

Gierliński, M., Zdziarski, A. A., Johnson, W. N., et al. 1996, Proc. Röntgenstrahlung from the Universe, ed. H. U. Zimmermann, J. Trümper, \& H. Yorke; MPE Report 263, 139

Gierliński, M., Zdziarski, A. A., Done, C., et al. 1997, MNRAS, 288, 958

Gierliński, M., Zdziarski, A. A, Poutanen, J., et al. 1999, MNRAS, 309,496

Gleissner, T., Wilms, J., Pottschmidt, K., et al. 2004a, A\&A, 414, 1091

Gleissner, T., Wilms, J., Pooley, G. G., et al. 2004b, A\&A, 425, 1061

Goldwurm, A., David, P., Foschini, L., et al. 2003, A\&A, 411, L223

Heinz, S., \& Sunyaev, R. A. 2003, MNRAS, 343, 59

Ibragimov, A., Poutanen, J., Gilfanov, M., et al. 2005, MNRAS, submitted [arXiv:astro-ph/0502423]

Jensen, P.-L., Clausen, K., Cassi, C., et al. 2003, A\&A, L7

Jourdain, E., \& Roques, J.-P. 1994, ApJ, 426, L11

Laurent, P., \& Titarchuk, L. G. 1999, ApJ, 511, 289

Lebrun, F., Leray, J. P., Lavocat, P., et al. 2003, A\&A, 411, L141

Ling, J. C., Wheaton, W. A., Wallyn, P., et al. 1997, ApJ, 484, 375

Lund, N., Butdtz-Jorgensen, C., Westergaard, N. L., et al. 2003, A\&A, 411, L231

Magdziarz, P., \& Zdziarski, A. A. 1995, MNRAS, 273, 837

Malzac, J., Petrucci, P. O., Jourdain, E., et al. 2004, Proceedings of the 5th INTEGRAL Workshop The INTEGRAL Universe, (Munich, February 2004), ESA Special Publication SP-552, [arXiv: astro-ph/0411069]

Malzac, J., Petrucci, P. O., Jourdain, E., et al. 2005, A\&A, submitted 
Markoff, S., Falcke, H., \& Fender, R. 2001, A\&A, 372, L25

Markoff, S., \& Nowak, M. A. 2004, ApJ, 609, 972

McClintock, J. E., \& Remillard, R. A. 2003, to appear in Compact Stellar X-ray Sources, ed. W. H. G. Lewin \& M. van der Klis [arXiv:astro-ph/0306213]

McConnell, M. L., Forrest, D., Ryan, J., et al. 1994, ApJ, 424, 933

McConnell, M. L., Ryan, J. M., Collmar, W., et al. 2000, ApJ, 543, 928

McConnell, M. L., Zdziarski, A. A., Bennett, K., et al. 2002, ApJ, 572, 984

Méndez, M., \& van der Klis, M., 1997, ApJ, 479, 926

Merloni, A., Fabian, A. C., \& Ross, R. R. 2000, MNRAS, 313, 193

Merloni, A., Heinz, S., \& di Matteo, T. 2003, MNRAS, 345, 1057

Misra, R., \& Melia, F. 1996, ApJ, 467, 405

Mitsuda, K., Inoue, H., Koyama, K., et al. 1984, PASJ, 36, 741

Nowak, M. A., 2002, in New Views on Microquasars, ed. P. Durouchoux, Y. Fuchs, \& J. Rodriguez (Kolbata: Centre for space Physics), 11

Nowak, M. A., Wilms, J., Heinz, S., et al. 2005, ApJ, 626, 1006

Pottschmidt, K., Wilms, J., Nowak, M. A., et al. 2003a, A\&A, 407, 1039

Pottschmidt, K., Wilms, J., Chernyakova, M., et al. 2003b, A\&A, 411, L383

Pottschmidt, K., Wilms, J., Nowak, M. A., et al. 2005, accepted for publication in the Proceedings of the 35th COSPAR Scientific Assembly, ASR publication [arXiv:astro-ph/0504403]

Poutanen, J., \& Svensson, R. 1996, ApJ, 470, 249

Poutanen, J. 1998, in Theory of Black Hole Accretion Discs, ed. M. A. Abramowicz, G. Bjornsson, \& J. E. Pringle (Cambridge: Cambridge Univ. Press), 100

Poutanen, J., \& Coppi, P. 1998, Phys. Scr., 77, 57 [arXiv:astro-ph/9711316]
Protassov, R., van Dyk, D., Connors, A., et al. 2002, ApJ, 571, 545

Rodriguez, J., Corbel, S., \& Tomsick, J. A. 2003, ApJ, 595, 1032

Roques, J.-P., Schanne, S., von Kienlin, A., et al. 2003, A\&A, 411, L91

Salotti, L., Ballet, J., Cordier, B., et al. 1992 A\&A, 253, 145

Shakura, N. I., \& Sunyaev, R. A. 1973, A\&A, 24, 337

Shimura, T., \& Takahara, F. 1995, ApJ, 445, 780

Skibo, J. G., \& Dermer, C. D., 1995, ApJ, 455, L25

Skinner, G., \& Connell, P. 2003, A\&A, 411, L123-126

Stirling, A., Spencer, R. E., de la Force, C. J., et al. 2001, MNRAS, 327,1273

Sunyaev, R. A., \& Titarchuk, L. 1980, A\&A, 86, 21

Titarchuk, L. G. 1994, ApJ, 434, 570

Titarchuk, L. G., Mastichiadis, A., \& Kylafis, N. D. 1997, ApJ, 487, 834

Ubertini, P., Lebrun, F., Di Cocco, G., et al. 2003, A\&A, 411, L131

Vedrenne, G., Roques, J.-P., Schönfelder, V., et al. 2003, A\&A, 411, L63

Westergaard, N. J., Kretschmar, P., Oxborrow, C. A., et al. 2003, A\&A, 411, L257

Winkler, C., Courvoisier, T. J.-L., Di Cocco, G., et al. 2003, A\&A, 411, L1

Zdziarski, A. A., Grove, J. E., Poutanen, J., Rao, A. R., \& Vadawale, S. V. 2001, ApJ, 554, L45

Zdziarski, A. A., Poutanen, J., Paciesas, W. S., \& Wen, L. 2002, ApJ, 578, 357

Zdziarski, A. A., \& Gierliński, M. 2004a, Progr. Theor. Phys. Suppl., 155, 99

Zdziarski, A. A., Gierliński, M., Mikolajewska, J., et al. 2004b, MNRAS, 351, 791

Ziólkowski, J., 2005, MNRAS, 358, 851 